Vietnam Journal of Mechanics, VAST, Vol.37, No. 1 (2015), pp. $43-56$

DOI:10.15625/0866-7136/37/1/5508

\title{
DYNAMIC STIFFNESS METHOD FOR FREE VIBRATIONS ANALYSIS OF PARTIAL FLUID-FILLED ORTHOTROPIC CIRCULAR CYLINDRICAL SHELLS
}

\author{
Tran Ich Thinh*, Nguyen Manh Cuong, Vu Quoc Hien \\ Hanoi University of Science and Technology, Vietnam \\ *E-mail: tranichthinh@yahoo.com \\ Received November 20, 2014
}

\begin{abstract}
Free vibrations of partial fluid-filled orthotropic circular cylindrical shells are investigated using the Dynamic Stiffness Method (DSM) or Continuous Element Method (CEM) based on the First Order Shear Deformation Theory (FSDT) and non-viscous incompressible fluid equations. Numerical examples are given for analyzing natural frequencies and harmonic responses of cylindrical shells partially and completely filled with fluid under various boundary conditions. The vibration frequencies for different filling ratios of cylindrical shells are obtained and compared with existing experimental and theoretical results which indicate that the fluid filling can reduce significantly the natural frequencies of studied cylindrical shells. Detailed parametric analysis is carried out to show the effects of some geometrical and material parameters on the natural frequencies of orthotropic cylindrical shells. The advantages of this current solution consist in fast convergence, low computational cost and high precision validating for all frequency ranges.

Keywords: Free vibration, continuous element method, dynamic stiffness method, orthotropic cylindrical shell, fluid-shell interaction.
\end{abstract}

\section{INTRODUCTION}

The knowledge on dynamic responses of fluid-filled isotropic and orthotropic cylindrical shells is of primary importance for the design of pressure vessels, fluid tanks of propellant rockets, seismic effects on liquid storage tanks etc. For coupled fluid-cylindrical shell problems, Jain [1] obtained the natural frequency equations of simply supported orthotropic cylindrical shells, filled completely or partially with an incompressible nonviscous fluid on the basis of a shell theory in which the effects of transverse shear deformation and rotatory inertia were retained. Warburton and Soni [2] studied the resonant response of orthotropic cylindrical shells, while Bradford and Dong [3] investigated the lateral vibrations of orthotropic cylinders under initial stress. The free vibrations of orthotropic cylindrical shells based on the three-dimensional elasticity theory considering

(C) 2015 Vietnam Academy of Science and Technology 
the effect of internal fluid have analyzed by Chen et al. [4] who obtained the frequency equation of non-axisymmetric free vibration modes of an orthotropic fluid-filled cylindrical shell with arbitrary constant thickness and then compared their results with those based on other shell theories. Li and Chen [5], basing on the Flugge's linear shell theory and the normal mode theory, study the dynamic response of orthotropic circular cylindrical shells subjected to external hydrostatic pressure. The effect of shell parameters, external hydrostatic pressure and material properties on the dynamic behavior of the shell has been examined in detail in their work. Using the Sanders-Koiter non-linear shell theory, Selmane and Lakis [6] investigated the influence of non-linearities associated with the shell wall and with the fluid flow on the dynamics of elastic thin orthotropic cylindrical shells using a hybrid finite element method. On the basis of the Ritz variation method, Shang and Lei [7] investigated the free vibrations of fluid-conveying orthotropic cylindrical shell. Sharma et al. [8] have investigated the natural frequency response of vertical cantilevered composite shells containing fluid. They used the Fourier series of trigonometric functions to approximate the axial modal dependence. This presents an example of practical applications of cylindrical shells. Recently, Prado et al. [9] studied non-linear vibrations and instabilities of orthotropic cylindrical shells with internal flowing fluid using the Galerkin approach. Daneshjou et al. [10] have proposed an analytical solution to solve the problem of sound transmission through orthotropic cylindrical shells with subsonic external flow. A boundary integral solution has been applied to the hydroelastic analysis of fluid storage tanks. Zhang et al. [11] have employed the wave propagation approach to investigate vibration characteristics of fluid-filled cylindrical shells. This gives relatively more accurate predictions of natural frequencies of cylindrical shells. Further showing the efficiency and efficacy of the wave propagation approach, Li et al. [12] have applied this technique to study the modal analysis of thin finite cylindrical shells. By the same method, Natsuki et al. [13] have carried out the vibrational analysis of fluidfilled carbon nanotube. This approach seems to be widely applicable to study vibration characteristics of shells involving fluids.

In those studies, low natural frequencies are generally investigated. For medium and high frequency range, the CEM can be applied with many advantages: high precision, rapid calculating speed, reduction of the model size and of the computing time. Numerous CEM researches have been performed for isotropic and composite beams: [14,15], plates: $[16,17]$ and shells: [18-20]. However, no work based on CEM appears to have been done on the problem of cylindrical shells taking into account the interaction of fluidstructure.

The purpose of this article is to present a new continuous element for isotropic and orthotropic cylindrical shells based on the Reissner-Mindlin shell theory which retains the effects of transverse shear deformation and rotatory inertia as well as those of nonviscous incompressible fluid. Numerical results indicate that the fluid filling can reduce significantly the natural frequencies of cylindrical shells. Parametric studies including circumferential wave number, fluid depth, thickness to mean radius ratio, length to mean radius and boundary condition are carried out. 


\section{THEORETICAL FORMULATION OF ORTHOTROPIC FLUID-FILLED CYLINDRICAL SHELLS}

\subsection{Kinematics of ortrotropic cylindrical shell filled with fluid}

Consider an ortrotropic cylindrical shell containing fluid as shown in Fig. 1. $R, L$ and $h$ are the radius, length and thickness of the cylinder. $H$ is the height of the contained fluid volume.

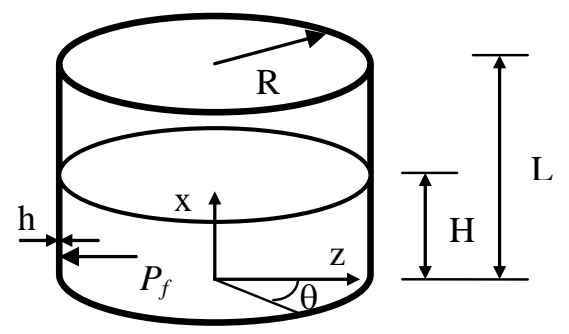

Fig. 1. Kinematics of orthotropic cylindrical shell filled with fluid

The material constants $Q_{i j}$ are defined in terms of the orthotropic material properties by

$$
\begin{array}{ll}
Q_{11}=E_{1} /\left(1-v_{12} v_{21}\right), & Q_{12}=v_{12} E_{2} /\left(1-v_{12} v_{21}\right), \\
Q_{22}=E_{2} /\left(1-v_{12} v_{21}\right), & Q_{66}=G_{12}, \quad Q_{44}=G_{23}, \quad Q_{55}=G_{13},
\end{array}
$$

where $E_{i}, G_{i j}, v_{12}, v_{21}$ : elastic constants of the material and the stiffness coefficients $\left(A_{i j}, D_{i j}\right)$ are

$$
\begin{aligned}
& A_{i j}=Q_{i j} h, \quad D_{i j}=Q_{i j} h^{3} / 12 \quad(i, j=1,2,6), \\
& A_{44}=h Q_{44}, \quad A_{55}=h Q_{55}, \quad A_{16}=A_{26}=A_{45}=0, \quad D_{16}=D_{26}=0 .
\end{aligned}
$$

Following Reissner-Mindlin assumption, the displacement components are assumed to be

$$
\begin{aligned}
& u(x, \theta, z, t)=u_{0}(x, \theta, t)+z \varphi_{x}(x, \theta, t), \\
& v(x, \theta, z, t)=v_{0}(x, \theta, t)+z \varphi_{\theta}(x, \theta, t), \\
& w(x, \theta, z, t)=w_{0}(x, \theta, t),
\end{aligned}
$$

where $u, v$ and $w$ are the displacement components in the $x, \theta$ and $z$ directions respectively; $u_{0}, v_{0}$ and $w_{0}$ are the displacements of the shell at the neutral surface in axial, circumferential and radial directions, and $\varphi_{x}$ and $\varphi_{\theta}$ are the rotations of the normal to the middle surface of the shell. The strain-displacement relations of cylindrical shell can be written as

$$
\begin{aligned}
& \varepsilon_{x}=\frac{\partial u_{0}}{\partial x}+z \frac{\partial \varphi_{x}}{\partial x}, \quad \varepsilon_{\theta}=\frac{1}{R} \frac{\partial v_{0}}{\partial \theta}+\frac{z}{R} \frac{\partial \varphi_{\theta}}{\partial \theta}+\frac{w_{0}}{R}, \\
& \gamma_{x \theta}=\frac{1}{R} \frac{\partial u_{0}}{\partial \theta}+\frac{\partial v_{0}}{\partial x}+z\left(\frac{1}{R} \frac{\partial \varphi_{x}}{\partial \theta}+\frac{\partial \varphi_{\theta}}{\partial x}\right), \\
& \gamma_{x z}=\varphi_{x}+\frac{\partial w_{0}}{\partial x}, \quad \gamma_{\theta z}=\varphi_{\theta}+\frac{1}{R} \frac{\partial w_{0}}{\partial \theta}-\frac{v_{0}}{R} .
\end{aligned}
$$




\subsection{Equations of motion for orthotropic cylindrical shells in contact with fluid}

The equations of motion of the first-order shear deformation theory for an orthotropic circular shell filled with fluid in $(x, \theta, z)$ coordinate are written by

$$
\begin{aligned}
& \frac{\partial N_{x}}{\partial x}+\frac{1}{R} \frac{\partial N_{\theta x}}{\partial \theta}=I_{0} \ddot{u}_{0}+I_{1} \ddot{\varphi}_{x}, \\
& \frac{\partial N_{x \theta}}{\partial x}+\frac{1}{R} \frac{\partial N_{\theta}}{\partial \theta}=I_{0} \ddot{v}_{0}+I_{1} \ddot{\varphi}_{\theta}, \\
& \frac{\partial Q_{x}}{\partial x}+\frac{1}{R} \frac{\partial Q_{\theta}}{\partial \theta}+\frac{1}{R} Q_{x}-P_{f}=I_{0} \ddot{w}_{0}, \\
& \frac{\partial M_{x}}{\partial x}+\frac{1}{R} \frac{\partial M_{\theta x}}{\partial \theta}-Q_{x}=I_{1} \ddot{u}_{0}+I_{2} \ddot{\varphi}_{x}, \\
& \frac{\partial M_{x \theta}}{\partial x}+\frac{1}{R} \frac{\partial M_{\theta}}{\partial \theta}-Q_{\theta}=I_{1} \ddot{v}_{0}+I_{2} \ddot{\varphi}_{\theta},
\end{aligned}
$$

where

$$
I_{i}=\int_{-h / 2}^{h / 2} \rho_{s} z^{i} \mathrm{~d} z, \quad(i=0,1,2),
$$

in which $\rho_{s}$ is the mass density of shell, $P_{f}$ is the hydrodynamic pressure of fluid which can be determined from fluid equations.

\subsection{Internal force resultants-displacement relationships}

Here, the internal force and moments resultants can be expressed in terms of displacements as [10]

$$
\begin{aligned}
& \left\{\begin{array}{l}
N_{x} \\
N_{\theta} \\
N_{\theta x} \\
N_{x \theta} \\
M_{x} \\
M_{\theta} \\
M_{\theta x} \\
M_{x \theta}
\end{array}\right\}=\left[\begin{array}{cccccccc}
A_{11} & A_{12} & 0 & 0 & \frac{D_{11}}{R} & 0 & 0 & 0 \\
A_{12} & A_{22} & 0 & 0 & 0 & -\frac{D_{22}}{R} & 0 & 0 \\
0 & 0 & A_{66} & A_{66} & 0 & 0 & \frac{D_{66}}{R} & 0 \\
0 & 0 & A_{66} & A_{66} & 0 & 0 & 0 & -\frac{D_{66}}{R} \\
\frac{D_{11}}{R} & 0 & 0 & 0 & D_{11} & D_{12} & 0 & 0 \\
0 & -\frac{D_{22}}{R} & 0 & 0 & D_{12} & D_{22} & 0 & 0 \\
0 & 0 & \frac{D_{66}}{R} & 0 & 0 & 0 & D_{66} & D_{66} \\
0 & 0 & 0 & -\frac{D_{66}}{R} & 0 & 0 & D_{66} & D_{66}
\end{array}\right]\left\{\begin{array}{c}
\frac{\partial u_{0}}{\partial x} \\
\frac{\partial v_{0}}{R \partial \theta}+\frac{w v_{0}}{R} \\
\frac{\partial v_{0}}{\partial x} \\
\frac{\partial u_{0}}{R \partial \theta} \\
\frac{\partial \varphi_{x}}{\partial x} \\
\frac{\partial \varphi_{\theta}}{R \partial \theta} \\
\frac{\partial \varphi_{\theta}}{\partial x} \\
\frac{\partial \varphi_{x}}{R \partial \theta}
\end{array}\right\}, \\
& \left\{\begin{array}{c}
Q_{x} \\
Q_{\theta}
\end{array}\right\}=k\left[\begin{array}{cc}
A_{55} & 0 \\
0 & A_{44}
\end{array}\right]\left\{\begin{array}{c}
\frac{\partial w_{0}}{\partial x}+\varphi_{x} \\
\frac{\partial w_{0}}{R \partial \theta}-\frac{v_{0}}{R}+\varphi_{\theta}
\end{array}\right\},
\end{aligned}
$$

where $k$ is the shear correction factor $(k=5 / 6)$.

\subsection{Fluid equations}

The cylindrical shell is partially or completely filled with an incompressible, inviscid liquid. For the steady-state case, the potential function $(r, \theta, x, t)$ satisfies the Laplace 
equation in cylindrical coordinates $(r, \theta, x)$

$$
\frac{\partial^{2} \Phi}{\partial r^{2}}+\frac{1}{r} \frac{\partial \Phi}{\partial r}+\frac{1}{r^{2}} \frac{\partial^{2} \Phi}{\partial \theta^{2}}+\frac{\partial^{2} \Phi}{\partial x^{2}}=0
$$

Then, the Bernoulli equation is written

$$
\frac{\partial \Phi}{\partial t}+\frac{P_{f}}{\rho_{f}}=0
$$

By linearizing this expression, the pressures on the internal regions are

$$
P_{f}=-\left.\rho \frac{\partial \Phi}{\partial t}\right|_{\Sigma} .
$$

The condition of impermeability of the surface of shell in contact with fluid may be expressed as

$$
v_{f}=\left.\frac{\partial \Phi}{\partial r}\right|_{\Sigma}=\left.\frac{\partial w_{0}}{\partial t}\right|_{\Sigma}
$$

where $w$ is the normal displacement of the shell, $v_{f}$ is the velocity of fluid.

The potential function $\Phi$ may be defined by the following expression

$$
\Phi(r, \theta, x, t)=\Psi(r) f(x) \cos (m \theta) e^{i \omega t},
$$

where $\Psi(r)$ is a function to be defined. Substitution (12) into Laplace's equation (8), gives

$$
\frac{\partial^{2} \Psi}{\partial r^{2}}+\frac{1}{r} \frac{\partial \Psi}{\partial r}-\left(\frac{m^{2}}{r^{2}}+k_{n}^{2}\right) \Psi=0 .
$$

Eq. (13) may be resolved using modified Bessel functions of first and second kind of order $m$, yielding

$$
\Psi(r)=A_{1} I_{m}\left(k_{n} r\right)+A_{2} K_{m}\left(k_{n} r\right) .
$$

Finally, the potential $\Phi$ is expressed as

$$
\Phi(r, \theta, x, t)=\left.\frac{\Psi(r)}{\Psi^{\prime}(r)} \frac{\partial w}{\partial t}\right|_{r=R} .
$$

And then the pressure $P$ is written by

$$
P_{f}(r, \theta, x, t)=-\left.\rho_{f} \frac{\Psi(r)}{\Psi^{\prime}(r)} \frac{\partial^{2} w}{\partial t^{2}}\right|_{r=R} .
$$

In addition, the pressure $P_{f}$ must remain finite, which means that the function $R(r)$ is defined by: $\Psi(r)=A_{1} I_{m}\left(k_{n} r\right)$ inside the shells.

The hydrodynamic pressure acting on the cylindrical shell is then defined by [21]

$$
P_{f}=-\rho_{f} \frac{1}{m+k_{n} R I_{m+1}\left(k_{n} R\right) / I_{m}\left(k_{n} R\right)} \frac{\partial^{2} w_{0}}{\partial t^{2}} .
$$

This value will be introduced in (5) in order to construct the Dynamic Stiffness Matrix for the studied structure. 


\section{CONTINUOUS ELEMENT FORMULATION FOR ORTHOTROPIC CYLINDRICAL SHELLS COMPLETELY FILLED WITH FLUID}

The state-solution vector is $\mathbf{y}=\left\{u_{0}, v_{0}, w_{0}, \varphi_{x}, \varphi_{\theta}, N_{x}, N_{x \theta}, Q_{x}, M_{x}, M_{x \theta}\right\}^{T}$. This vector can be expressed in the Fourier expansion form, for the symmetric circumferential mode $m$ as

$$
\begin{aligned}
& \left\{u_{0}(x, \theta, t) \quad w_{0}(x, \theta, t) \quad \varphi_{x}(x, \theta, t) \quad N_{x}(x, \theta, t) \quad Q_{x}(x, \theta, t) \quad M_{x}(x, \theta, t)\right\}^{T}=
\end{aligned}
$$

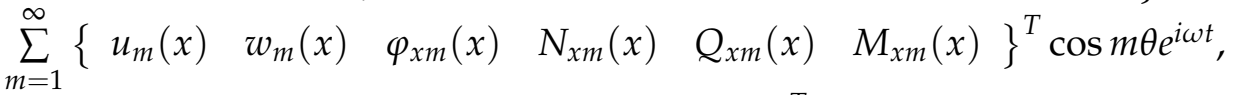

$$
\begin{aligned}
& \left\{\begin{array}{llll}
v_{0}(x, \theta, t) & N_{x \theta}(x, \theta, t) & \varphi_{\theta}(x, \theta, t) & M_{x \theta}(x, \theta, t)
\end{array}\right\}^{T}= \\
& \sum_{m=1}^{\infty}\left\{\begin{array}{llll}
v_{m}(x) & N_{x \theta m}(x) & \varphi_{\theta m}(x) & M_{x \theta m}(x)
\end{array}\right\}^{T} \sin m \theta e^{i \omega t} .
\end{aligned}
$$

Similar transformations for anti-symmetric modes will easily be obtained by swapping $\cos (m \theta)$ and $\sin (m \theta)$ in (18). Further detailed formulations in this research concern only symmetric modes for the sake of simplification.

Applying the CEM procedure presented in $[16,20]$, the following differential equations have been calculated

$$
\begin{aligned}
\frac{\mathrm{d} w_{m}}{\mathrm{~d} x}= & \frac{1}{k A_{55}} Q_{x m}-\varphi_{x m}, \frac{d \varphi_{x m}}{\mathrm{~d} x}=-\frac{1}{c_{1}} N_{x m}-\frac{c_{5}}{c_{1} c_{3}} M_{x m}-c_{4} w_{m}-m c_{4} v_{m}+c_{5} m \varphi_{\theta m}, \\
\frac{\mathrm{d} \varphi_{\theta m}}{\mathrm{~d} x}= & \frac{B_{66}}{c_{6}} N_{x \theta m}-\frac{A_{66}}{c_{6}} M_{x \theta m}-m \varphi_{x m}-\frac{1}{R} \varphi_{\theta m}, \\
\frac{\mathrm{d} N_{x m}}{\mathrm{~d} x}= & \frac{1}{R}\left(\frac{c_{5}}{c_{1}}-1\right) N_{x m}+\frac{c_{4}}{c_{1} R} M_{x m}+\left(I_{0} \omega^{2}+\frac{A_{12} c_{2}}{c_{1} R^{2}}-\frac{B_{12} c_{4}}{c_{1} R^{2}}\right) u_{m}+ \\
& \frac{m c_{7}}{R^{2}} v_{m}-\frac{m}{R} N_{x \theta m}+\left(I_{1} \omega^{2}+\frac{c_{8}}{R^{2}}\right) \varphi_{x m}+\frac{c_{8} m}{R^{2}} \varphi_{\theta m}, \\
\frac{\mathrm{d} N_{x \theta m}}{\mathrm{~d} x}= & \frac{m c_{5}}{R c_{1}} N_{x m}+\frac{m c_{4}}{c_{1} R} M_{x m}-\frac{m}{R^{2}}\left(A_{11} \frac{c_{2}}{c_{1}}-B_{12} \frac{c_{4}}{c_{1}}\right) u_{m}+ \\
& \left(I_{0} \omega^{2}+\frac{m^{2} c_{7}}{R^{2}}\right) v_{m}-\frac{2}{R} N_{x \theta m}+\frac{m c_{8}}{R^{2}} \varphi_{x m}+\left(I_{1} \omega^{2}+\frac{m^{2} c_{8}}{R^{2}}\right) \varphi_{\theta m}, \\
\frac{\mathrm{d} Q_{x m}}{\mathrm{~d} x}= & \left(I_{0} \omega^{2}-p_{f} \omega^{2}+\frac{k A_{44} m^{2}}{R^{2}}\right) w_{m}-\frac{1}{R} Q_{x m}-\frac{k A_{44} m}{R} \varphi_{\theta m}, \\
\frac{\mathrm{d} M_{x m}}{\mathrm{~d} x}= & \frac{1}{R}\left(\frac{c_{5}}{c_{1}}-1\right) M_{x m}+\left(I_{1} \omega^{2}+\frac{c_{9}}{R^{2}}\right) u_{m}+\frac{m c_{9}}{R^{2}} v_{m}+Q_{x m}-\frac{c_{3}}{c_{1} R} N_{x m}+ \\
& \left(I_{2} \omega^{2}+\frac{c_{10}}{R^{2}}\right) \varphi_{x m}+\frac{c_{10} m}{R^{2}} \varphi_{\theta m}-\frac{m}{R} M_{x \theta m}, \\
\frac{\mathrm{d} M_{x \theta m}}{\mathrm{~d} x}= & \frac{m c_{5}}{R c_{1}} M_{x m}+\left(I_{1} \omega^{2}+\frac{c_{9} m^{2}}{R^{2}}\right) v_{m}+\frac{m c_{9}}{R^{2}} u_{m}-\frac{k A_{44} m}{R} w_{m}-\frac{m c_{3}}{c_{1} R} N_{x m}+ \\
& \frac{m c_{10}}{R^{2}} \varphi_{x m}+\left(I_{2} \omega^{2}+k A_{44}+\frac{c_{10} m^{2}}{R^{2}}\right) \varphi_{\theta m}-\frac{2}{R} M_{x \theta m}
\end{aligned}
$$


with

$$
\begin{aligned}
& c_{1}=-B_{11}^{2}+A_{11} D_{11}, c_{2}=\left(A_{12} B_{11}-A_{11} B_{12}\right) / c_{1}, c_{3}=\left(B_{11} B_{12}-A_{11} D_{12}\right) / c_{1}, \\
& c_{4}=\left(B_{12} B_{11}-A_{12} D_{11}\right) / c_{1}, c_{5}=\left(B_{11} D_{12}-B_{12} D_{11}\right) / c_{1}, c_{6}=A_{12} c_{4}+B_{12} c_{2}+A_{22}, \\
& c_{7}=A_{12} c_{5}+B_{12} c_{3}+B_{22}, c_{8}=B_{12} c_{4}+D_{12} c_{2}+B_{22}, c_{9}=B_{12} c_{5}+D_{22}+D_{12} c_{3}, \\
& c_{10}=B_{66}^{2}-A_{66} D_{66}, c_{11}=\left(A_{12} c_{3}+D_{22} / R^{2}\right) / R, c_{12}=\left(D_{12} c_{4}+D_{22} / R^{2}\right) / R .
\end{aligned}
$$

Eq. (19) can be expressed in the matrix form for each circumferential mode $m$

$$
\frac{\mathrm{d} \mathbf{y}_{m}}{\mathrm{~d} x}=\mathbf{A}_{m} \mathbf{y}_{m}
$$

where $A_{m}$ is a $10 \times 10$ matrix (see Appendix).

The dynamic transfer matrix $\mathbf{T}_{m}$ is evaluated by $[16,17]$

$$
\mathbf{T}_{m}=e^{\mathbf{A}_{m} L}=\left[\begin{array}{ll}
\mathbf{T}_{11} & \mathbf{T}_{12} \\
\mathbf{T}_{21} & \mathbf{T}_{22}
\end{array}\right]_{m}
$$

And the dynamic stiffness matrix $\mathbf{K}(\omega)_{m}$ is $[17,18,20]$

$$
\mathbf{K}(\omega)_{m}=\left[\begin{array}{cc}
\mathbf{T}_{12}^{-1} \mathbf{T}_{11} & -\mathbf{T}_{12}^{-1} \\
\mathbf{T}_{21}-\mathbf{T}_{22} \mathbf{T}_{12}^{-1} \mathbf{T}_{11} & \mathbf{T}_{22} \mathbf{T}_{12}^{-1}
\end{array}\right]_{m}
$$

\section{ASSEMBLY PROCEDURE FOR CONTINUOUS ELEMENTS OF PARTIALLY FLUID-FILLED CYLINDRICAL SHELLS}

It is important to note that the proposed Dynamic Stiffness Matrix can be used for both orthotropic cylinders without fluid (by setting $\rho_{f}=0$ ) and for cylindrical shells completely filled with fluid $\left(\rho_{f} \neq 0\right)$.

Naturally, a partially fluid-filled cylinder is composed by two regions with different characteristics of material and geometry: an empty zone and a fluid-contained one (see Fig. 2). The problem becomes complex which requires a much more complicated coupled fluid-shell system of equations to solve. In such case, the application of analytical approaches in order to resolve those system is obviously very tedious and difficult. Moreover, FEM simulation meets also difficulties either in modeling fluid-shell structures with various properties or in choosing an appropriate meshing for obtaining accurate solutions.

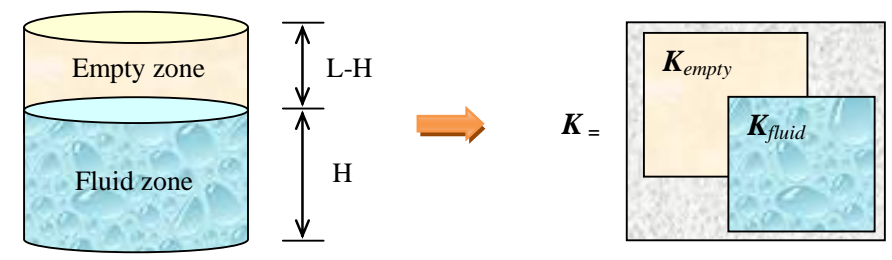

Fig. 2. Assembly of Dynamic Stiffness Matrix for a partially fluid-filled cylinder

The assembly technique of continuous elements demonstrates it's performance in this situation. That procedure is a powerful capacity of CEM and has successfully been 
applied in our previous research [17] to study the hard problem of composite plates resting on non-homogenous elastic foundation. The logical development of continuous element model consists to adapt this assembly algorithm to the problem of partially fluidfilled cylindrical shells.

Fig. 2 illustrates the construction of the Dynamic Stiffness Matrix for a partial fluidfilled cylindrical shell using the assembly procedure. Despite the complexity of the problem, the proposed algorithm makes possible a simple and efficient solution. In this case, the different mentioned zones of the shell are modeled by two Dynamic Stiffness Matrices i.e.: $\mathbf{K}_{\text {empty }}(\omega)$ for the empty region (with $\rho_{f}=0$ ) and $\mathbf{K}_{\text {fluid }}(\omega)$ for the completely fluid-filled zone $\left(\rho_{f} \neq 0\right)$. Finally, the Dynamic Stiffness Matrix of the partially fluidfilled cylinder will be obtained by an assembly of the two above matrices similarly to the method used in our previous researches [16-18,20]. This strong capacity of CEM consists an interesting and powerful approach to deal with complicated structures such as shell partially in contact with inside or outside fluid, combined conical-cylindrical shells, shells with stiffeners ...

\section{NUMERICAL RESULTS AND DISCUSSION}

A Matlab program has been written using the presented formulation in order to study the vibrational behavior of orthotropic cylindrical shells in contact with fluid and subjected to various boundary conditions. Obtained values will be validated by comparing with available results in the literature and with FEM.

\subsection{Free vibration of orthotropic cylindrical shell filled with fluid}

Now our model will be validated for orthotropic cylindrical shell filled with fluid. The cylinder is subjected to simply supported-simply supported boundary condition. The following dimensionless natural frequency is used for comparison: $\Omega=\omega R \sqrt{\left(1-v_{12} v_{21}\right) \rho / E_{1}}$.

A parametric analysis for orthotropic cylindrical shells without fluid has been conducted using five different cases of orthotropic material with varying $E_{1} / E_{2}$ relations and Poisson ratios. All studied orthotropic cases are resumed in Tab. 1. Only the first longitudinal mode is considered in Tab. 2 and Tab. 3.

Table 1. Shell material properties

\begin{tabular}{|c|c|c|c|c|c|}
\hline Case & $v_{12}$ & $v_{21}$ & $E_{1}(\mathrm{GPa})$ & $E_{2}(\mathrm{GPa})$ & $G_{12}(\mathrm{GPa})$ \\
\hline 1 & 0.131926 & 0.012114 & 227.350 & 20.876 & 7.958 \\
\hline 2 & 0.131926 & 0.04 & 68.599 & 20.799 & 7.958 \\
\hline 3 & 0.131926 & 0.131926 & 20.545 & 20.545 & 7.958 \\
\hline
\end{tabular}

Obtained results by CEM comparing to those of Warburton and Soni [2] and Li and Chen [5] basing on theirs analytical solution are illustrated in Tab. 2. For circumferential modes varying from 2 to 7 , the high precision of our model is confirmed. The present 
Dynamic stiffness method for free vibrations analysis of partial fluid-filled orthotropic circular ...

Table 2. Comparison of natural frequency parameter $\Omega$ for the orthotropic cylindrical shell with published results. $L / R=2.0, h / R=0.01$ and $m=1$

\begin{tabular}{|c|c|c|c|c|c|c|c|}
\hline \multirow{2}{*}{} & \multirow{2}{*}{ Case } & \multicolumn{7}{|c|}{$n$} \\
\cline { 3 - 8 } & & 2 & 3 & 4 & 5 & 6 & 7 \\
\hline \multirow{2}{*}{$\begin{array}{c}\text { Warburton } \\
\text { and Soni } \\
{[2]}\end{array}$} & 1 & 0.119875 & 0.085272 & 0.065184 & 0.054311 & 0.050975 & 0.054227 \\
\cline { 2 - 8 } & 2 & 0.207798 & 0.139463 & 0.100747 & 0.081915 & 0.079310 & 0.089331 \\
\cline { 2 - 8 } Li and & 3 & 0.330057 & 0.198610 & 0.134684 & 0.113414 & 0.122501 & 0.150636 \\
\cline { 2 - 8 } Chen [5] & 1 & 0.119888 & 0.085277 & 0.065190 & 0.054336 & 0.051014 & 0.054286 \\
\cline { 2 - 8 } & 3 & 0.207822 & 0.139494 & 0.100772 & 0.081952 & 0.079355 & 0.089433 \\
\hline \multirow{3}{*}{ Present } & 1 & 0.330100 & 0.198607 & 0.134742 & 0.113515 & 0.122652 & 0.150753 \\
\cline { 2 - 8 } & 2 & 0.2078 & 0.1395 & 0.1007 & 0.0819 & 0.0793 & 0.0893 \\
\cline { 2 - 8 } & 3 & 0.3301 & 0.1986 & 0.1346 & 0.1134 & 0.1225 & 0.1506 \\
\hline
\end{tabular}

Table 3. Natural frequency parameter $\Omega$ for the fluid-filled orthotropic cylindrical shell and varying $n ; L / R=1.0, h / R=0.01$ and $m=1$

\begin{tabular}{|c|c|c|c|c|c|c|c|c|}
\hline \multirow{2}{*}{ Case } & \multicolumn{9}{|c|}{$n$} \\
\cline { 2 - 9 } & \multicolumn{2}{|c|}{5} & \multicolumn{2}{|c|}{6} & \multicolumn{2}{|c|}{7} & \multicolumn{2}{|c|}{8} \\
\cline { 2 - 9 } & $\begin{array}{c}\text { Prado } \\
{[9]}\end{array}$ & Present & $\begin{array}{c}\text { Prado } \\
{[9]}\end{array}$ & Present & $\begin{array}{c}\text { Prado } \\
{[9]}\end{array}$ & Present & $\begin{array}{c}\text { Prado } \\
{[9]}\end{array}$ & Present \\
\hline 1 & 0.0628 & 0.0629 & 0.0585 & 0.0586 & 0.0575 & 0.0575 & 0.0597 & 0.0597 \\
\hline 2 & 0.1049 & 0.1049 & 0.0954 & 0.0953 & 0.0929 & 0.0926 & 0.0975 & 0.0971 \\
\hline 3 & 0.1616 & 0.1601 & 0.1437 & 0.1422 & 0.1426 & 0.1408 & 0.1568 & 0.1546 \\
\hline
\end{tabular}

formulation offers good values which are perfectly closed to those of $\mathrm{Li}$ and Chen. And this similitude is observed for three investigated cases of orthotropic materials.

The last analysis consists of a simply supported orthotropic cylindrical shell filled with water. In Tab. 3, natural frequencies of a cylinder $(L / R=1.0, h / R=0.01)$ with five above orthotropic materials are computed and compared to work of Prado et al. [9] using their analytical solutions.

It is obvious to remark very small differences between results calculated by CEM and by Prado et al. [9]. Moreover, the high precision of our model are validated through out all five cases of orthotropic materials. Therefore, by using the exact closed form solution of the differential equations of the structure, CEM using only two elements is an attractive approach with high precision and small volume of data storage, especially for analyzing vibrations of isotropic and orthotropic cylindrical shells partially or fully filled 
with water. Otherwise, these advantages combining with the possibility of using the assembly procedure of FEM are precious features of CEM which allows our formulation working well in all low, medium and high frequency ranges.

\subsection{Effect of filled fluid level and shells parameters on frequency of orthotropic cylin- drical shells}

First, in this section, the effect of filled fluid level on frequency of clamped-free orthotropic cylindrical shells is investigated. The dimensions of cylindrical shell and material properties are: $E_{1}=227.35 \mathrm{GPa} ; E_{2}=20.87 \mathrm{GPa} ; v_{12}=0.131926 ; G_{12}=G_{13}=$ $G_{23}=7.958 \mathrm{GPa} ; h=0.0254 \mathrm{~m} ; R=10 h ; L=2 R ; \rho_{s}=1600 \mathrm{~kg} / \mathrm{m}^{3} ; \rho_{f}=1000 \mathrm{~kg} / \mathrm{m}^{3}$. Tab. 4 shows the influence of the liquid level on the natural frequencies with corresponding modes.

Table 4. Effect of fluid level on natural frequencies $(\mathrm{Hz})$ of a clamped-free orthotropic cylindrical shell

\begin{tabular}{|l|c|c|c|c|c|c|}
\hline \multirow{3}{*}{$m$} & $n$ & \multicolumn{5}{|c|}{$H / L$} \\
\cline { 3 - 7 } & & 0 & 0.25 & 0.5 & 0.75 & 1 \\
\hline \multirow{4}{*}{1} & 1 & 830.97 & 829.23 & 784.92 & 649.29 & 429.40 \\
\cline { 2 - 7 } & 2 & 588.73 & 587.44 & 554.96 & 457.04 & 241.82 \\
\cline { 2 - 7 } & 3 & 663.56 & 662.34 & 630.01 & 530.09 & 357.04 \\
\cline { 2 - 7 } & 4 & 1021.44 & 1019.59 & 968.78 & 827.74 & 653.63 \\
\cline { 2 - 7 } & 5 & 1549.77 & 1546.60 & 1453.77 & 1258.47 & 1067.59 \\
\hline \multirow{4}{*}{2} & 1 & 2090.28 & 2045.59 & 1669.42 & 1501.30 & 952.53 \\
\cline { 2 - 7 } & 2 & 1722.11 & 1682.59 & 1388.27 & 1279.81 & 728.20 \\
\cline { 2 - 7 } & 3 & 1621.99 & 1584.02 & 1319.88 & 1237.40 & 706.47 \\
\cline { 2 - 7 } & 4 & 1782.03 & 1739.55 & 1477.01 & 1410.59 & 921.49 \\
\cline { 2 - 7 } & 5 & 2161.95 & 2108.57 & 1838.39 & 1780.29 & 1307.25 \\
\hline \multirow{4}{*}{3} & 1 & 3303.99 & 3153.33 & 2767.53 & 2162.56 & 1183.10 \\
\cline { 2 - 7 } & 2 & 3233.23 & 3012.01 & 2673.74 & 2198.16 & 1196.37 \\
\cline { 2 - 7 } & 3 & 3190.24 & 2971.26 & 2671.77 & 2267.92 & 1244.81 \\
\cline { 2 - 7 } & 4 & 3289.25 & 3067.29 & 2798.34 & 2435.18 & 1432.81 \\
\cline { 2 - 7 } & 5 & 3539.30 & 3307.88 & 3061.84 & 2718.17 & 1782.48 \\
\hline
\end{tabular}

The results in Tab. 4 show that, for all the modes of vibration considered, the filled fluid can reduce significantly the natural frequency of an orthotropic cylindrical shell. For example, for the first mode $(m=1, n=1)$, the natural frequency is approximately $0.25 \%, 5.5 \%, 21.8 \%$ and $48.3 \%$ corresponding to fluid filling levels, $H / L=0.25,0.5,0.75$ and 1.0 respectively lower in fluid than in air $(H / L=0)$, whereas for the mode $m=$ $3, n=3$, the reduction of the natural frequency is approximately $6.8 \%, 16.3 \%, 28.9 \%$ and $61 \%$ corresponding to fluid filling levels, $H / L=0.25,0.5,0.75$ and 1.0 respectively. 
Consequently, the high modes are more sensitive to the effect of the containing fluid than the low modes. From the Tab. 4, it can be seen clearly that the reduction of the natural frequency of the cylindrical shell decreases first slowly then quickly as the fluid height increases.

Next, the effects of length-to radius ratio and thickness-to radius ratio on the frequencies of fluid-filled simply-supported orthotropic cylindrical shell are presented in Figs. 3-4, respectively.

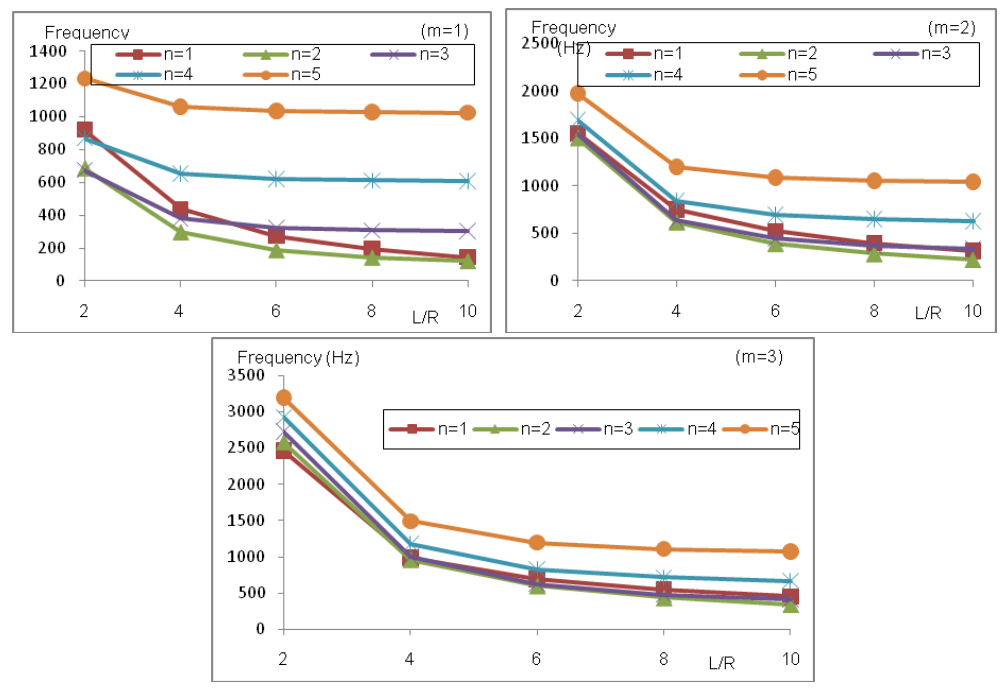

Fig. 3. Variation of natural frequencies $(\mathrm{Hz})$ with various values of $L / R$ for orthotropic fluid-filled simply supported cylindrical shell $(m=1,2,3)$

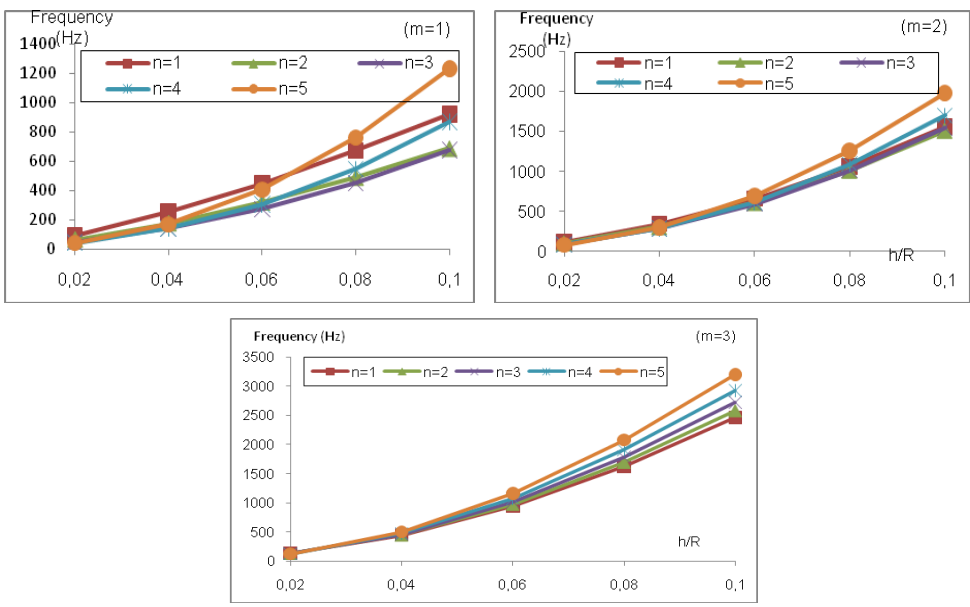

Fig. 4. Variation of natural frequencies $(\mathrm{Hz})$ with various values of $h / R$ for orthotropic fluid-filled simply supported-supported cylindrical shell $(m=1,2,3)$ 
Fig. 3 shows variations of natural frequencies of the orthotropic cylindrical with simply supported edges and completely filled with fluid, versus $L / R$ The values of natural frequencies of the cylindrical shells for all modes are decrease as the ratio, $L / R$, increase. For example, as the ratio, $L / R$, increases from 2 to 10, for the first mode $(m=1, n=1)$ the natural frequencies are decreased from $921.05 \mathrm{~Hz}$ to $144.6 \mathrm{~Hz}$; for the mode $m=3, n=3$, the natural frequencies are decreased from $2725.76 \mathrm{~Hz}$ to $409.48 \mathrm{~Hz}$, etc.

The effect of the variation of the ratio, $h / R$, on the natural frequencies of orthotropic shells with simply supported edges and completely filled with fluid is illustrated in Fig. 4 The values of natural frequencies of orthotropic cylindrical shells are increased, as the ratio, $h / R$, increase. For example, as the ratio, $h / R$, increases from 0.02 to 0.1 , for the first mode $(m=1, n=1)$ the natural frequencies are increased from $93.36 \mathrm{~Hz}$ to $921.05 \mathrm{~Hz}$; for the third mode $(m=3, n=3)$, the natural frequencies are increased from $130.05 \mathrm{~Hz}$ to $2725.76 \mathrm{~Hz}$, etc.

\section{CONCLUSIONS}

A new continuous element for orthotropic cylindrical shell with or without contact with fluid has been successfully constructed in this research. Furthermore, a simple and robust assembly method has been introduced for analyzing the vibration characteristics of partially fluid-filled orthotropic cylindrical shells. Different test cases have been examined which confirm the validity of the proposed formulation. Excellent agreements are found between results by Continuous Element Method and by other published ones demonstrating the high precision of this model. Continuous Element solutions for the mentioned structure reveals strong influences of fluid on vibratoire behaviors of the shell. Fluid effects are expressed by reducing significantly the natural frequencies and by changing the mode shapes of the structure. Such phenomenon must be taken into account in designs of isotropic and orthotropic cylinders in contact with fluid. Advantages of the presented model are low computational cost and the possibility of application in medium and high frequency range. Those precious characteristics of Continuous Element Method are high-lighted in the case of fluid-structure interaction problem while the precision and computational time of Finite Element models are affected by the demand of a huge number of meshing elements.

\section{ACKNOWLEDGEMENTS}

This research is funded by Vietnam National Foundation for Science and Technology Development (NAFOSTED) under grant number: 107.02-2013.25.

\section{REFERENCES}

[1] R. K. Jain. Vibration of fluid-filled, orthotropic cylindrical shells. Journal of Sound and Vibration, 37, (3), (1974), pp. 379-388.

[2] G. B. Warburton and S. R. Soni. Resonant response of orthotropic cylindrical shells. Journal of Sound and Vibration, 53, (1), (1977), pp. 1-23.

[3] L. G. Bradford and S. B. Dong. Natural vibrations of orthotropic cylinders under initial stress. Journal of Sound and Vibration, 60, (2), (1978), pp. 157-175. 
[4] C. Weiqiu, H. J. Ding, Y. Guo, and Q. D. Yang. Free vibrations of fluid-filled orthotropic cylindrical shells. Journal of Engineering Mechanics, 123, (11), (1997), pp. 1130-1133.

[5] X. Li and Y. Chen. Transient dynamic response analysis of orthotropic circular cylindrical shell under external hydrostatic pressure. Journal of Sound and Vibration, 257, (5), (2002), pp. 967-976.

[6] A. Selmane and A. Lakis. Non-linear dynamic analysis of orthotropic open cylindrical shells subjected to a flowing fluid. Journal of Sound and Vibration, 202, (1), (1997), pp. 67-93.

[7] S. P. Shang and G. P. Lei. The free vibration of orthotropic moderately thick cylindrical shells conveying fluid. Applicatiolls of Mech. ill ElIgrg., Hangzhou, (1989), pp. 231-241.

[8] C. B. Sharma, M. Darvizeh, and A. Darvizeh. Natural frequency response of vertical cantilever composite shells containing fluid. Engineering Structures, 20, (8), (1998), pp. 732-737.

[9] Z. del Prado, P. B. Gonçalves, and M. P. Païdoussis. Non-linear vibrations and instabilities of orthotropic cylindrical shells with internal flowing fluid. International Journal of Mechanical Sciences, 52, (11), (2010), pp. 1437-1457.

[10] K. Daneshjou, A. Nouri, and R. Talebitooti. Analytical model of sound transmission through orthotropic cylindrical shells with subsonic external flow. Aerospace Science and Technology, 13, (1), (2009), pp. 18-26.

[11] X. M. Zhang, G. R. Liu, and K. Y. Lam. Coupled vibration analysis of fluid-filled cylindrical shells using the wave propagation approach. Applied Acoustics, 62, (3), (2001), pp. 229-243.

[12] L. I. Bing-Ru, W. Xuan-yin, G. E. Hui-liang, and D. Yuan-ming. Study on applicability of modal analysis of thin finite length cylindrical shells using wave propagation approach. Journal of Zhejiang University Science A, 6, (10), (2005), pp. 1122-1127.

[13] T. Natsuki, M. Endo, et al. Vibrational analysis of fluid-filled carbon nanotubes using the wave propagation approach. Applied Physics A, 90, (3), (2008), pp. 441-445.

[14] R. Lundén and B. Åkesson. Damped second-order Rayleigh-Timoshenko beam vibration in space-an exact complex dynamic member stiffness matrix. International Journal for Numerical Methods in Engineering, 19, (3), (1983), pp. 431-449.

[15] J. R. Banerjee. Development of an exact dynamic stiffness matrix for free vibration analysis of a twisted Timoshenko beam. Journal of Sound and Vibration, 270, (1), (2004), pp. 379-401.

[16] M. C. Nguyen. Elements continus de plaques et coques avec prise en compte du cisaillement transverse: application à l'interaction fluide-structure. PhD thesis, Paris 6, (2003).

[17] T. I. Thinh, M. C. Nguyen, and D. G. Ninh. Dynamic stiffness formulation for vibration analysis of thick composite plates resting on non-homogenous foundations. Composite Structures, 108, (2014), pp. 684-695.

[18] J. B. Casimir, M. C. Nguyen, and I. Tawfiq. Thick shells of revolution: Derivation of the dynamic stiffness matrix of continuous elements and application to a tested cylinder. Computers E Structures, 85, (23), (2007), pp. 1845-1857.

[19] M. A. Khadimallah, J. B. Casimir, M. Chafra, and H. Smaoui. Dynamic stiffness matrix of an axisymmetric shell and response to harmonic distributed loads. Computers $\mathcal{E}$ Structures, 89, (5), (2011), pp. 467-475.

[20] T. I. Thinh and M. C. Nguyen. Dynamic stiffness matrix of continuous element for vibration of thick cross-ply laminated composite cylindrical shells. Composite Structures, 98, (2013), pp. 93-102.

[21] M. P. Paidoussis and J. P. Denise. Flutter of thin cylindrical shells conveying fluid. Journal of Sound and Vibration, 20, (1), (1972), pp. 9-26. 


\section{APPENDIX}

$[\mathbf{A}]_{m}$ matrix expression:

$$
\begin{aligned}
& {\left[\begin{array}{cccc}
0 & m c_{2} & c_{2} & 0 \\
-m c_{7} & 0 & 0 & m c_{8} \\
0 & 0 & 0 & -1 \\
0 & m c_{4} & -c_{4} & 0 \\
0 & 0 & 0 & -m \\
I_{0} \omega^{2}+\frac{A_{12} c_{2}}{c_{1} R^{2}}-\frac{B_{12} c_{4}}{c_{1} R^{2}} & \frac{m c_{7}}{R^{2}} & 0 & I_{1} \omega^{2}+\frac{c_{8}}{R^{2}} \\
-\frac{m}{R^{2}}\left(A_{11} \frac{c_{2}}{C_{1}}-B_{12} \frac{c_{4}}{c_{1}}\right) & I_{0} \omega^{2}+\frac{m^{2} c_{7}}{R^{2}} & 0 & \frac{m c_{8}}{R^{2}} \\
0 & 0 & \left(I_{0}-p_{f}\right) \omega^{2}+\frac{m^{2} K A_{44}}{R^{2}} & 0 \\
\left(I_{1} \omega^{2}+\frac{c_{9}}{R^{2}}\right) & \frac{m c_{9}}{R^{2}} & 0 & \left(I_{2} \omega^{2}+\frac{c_{10}}{R^{2}}\right) \\
\frac{m c_{9}}{R^{2}} & I_{1} \omega^{2}+\frac{m^{2} c_{9}}{R^{2}} & -\frac{k A_{44} m}{R} & \frac{m c_{10}}{R^{2}}
\end{array}\right.} \\
& \begin{array}{llllll}
-m c_{3} & \frac{R}{c_{1}} & 0 & 0 & \frac{1}{c_{1}} & 0
\end{array} \\
& \begin{array}{llllll}
0 & 0 & -\frac{R}{c_{6}} & 0 & 0 & \frac{1}{c_{6}}
\end{array} \\
& \begin{array}{llllll}
0 & 0 & 0 & \frac{1}{k A_{55}} & 0 & 0
\end{array} \\
& \begin{array}{llllll}
m c_{5} & -\frac{1}{c_{1}} & 0 & 0 & \frac{c_{5}}{c_{3} c_{1}} & 0
\end{array} \\
& \begin{array}{cccccc}
-\frac{1}{R} & 0 & \frac{B_{66}}{c_{6}} & 0 & 0 & -\frac{A_{66}}{c_{6}}
\end{array}
\end{aligned}
$$

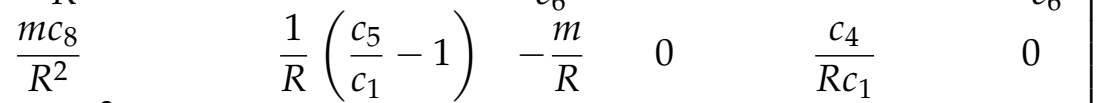

$$
\begin{aligned}
& \begin{array}{llllll}
I_{1} \omega^{2}+\frac{m^{2} c_{8}}{R^{2}} & \frac{m c_{5}}{R c_{1}} & -\frac{2}{R} & 0 & \frac{m c_{4}}{R c_{1}} & 0
\end{array} \\
& \begin{array}{cccccc}
-\frac{m k A_{44}}{R} & 0 & 0 & -\frac{1}{R} & 0 & 0
\end{array} \\
& \begin{array}{lllll}
\frac{m c_{10}}{R^{2}} & \frac{c_{3}}{c_{1} R} & 0 & 1 & \frac{1}{R}\left(\frac{c_{5}}{c_{1}}-1\right)
\end{array} \\
& -I_{2} \omega^{2}+K A_{44}+\frac{c_{10} m^{2}}{R^{2}} \quad-\frac{m c_{3}}{c_{1} R} \quad \begin{array}{lllll}
R & 0 & \frac{m c_{54}}{R c_{1}} & -\frac{2}{R}
\end{array}
\end{aligned}
$$

